\title{
La hormonoterapia combinada y la simvastatina mejoran el perfil lipídico en mujeres postmenopáusicas con hipercolesterolemia
}

Estrogen and progestin compared with simvastatin for hypercholesterolemia in postmenopausal women. Darling GM, Johns JA, Mc Cloud PI et al. N Engl J Med 1997;337:595-601

\section{Objetivo}

Comparar el tratamiento de estrógenos y progesterona (TRH) con el de simvastatina (SMV) en mujeres postmenopáusicas con hipercolesterolemia.

\section{Diseño}

Ensayo clínico randomizado, doble ciego, cruzado. Duración: 24 semanas.

Lugar

Melbourne, Australia.

\section{Pacientes}

Se incluyeron 58 mujeres postmenopáusicas con colesterol $>250 \mathrm{mg} / \mathrm{dl}$; mamografía y Papanicolau normales; que no hubieran recibido TRH o hipolipemiantes en las ocho semanas previas. Se excluyeron mujeres con antecedentes de cáncer estrógeno-dependiente, tromboembolismo, enfermedad cerebrovascular, hipertensión no controlada, me- trorragia sin diagnóstico, consumo de alcohol $>30$ g/día o hepatograma alterado.

Intervención

Cada mujer recibió SMV $10 \mathrm{mg} /$ día y TRH ( $5 \mathrm{mg} /$ día de medroxiprogesterona y dosis creciente de estrógenos conjugados equinos hasta llegar a $1.25 \mathrm{mg} /$ día) por 8 semanas, con un periodo de lavado entre los dos tratamientos de 8 semanas. La secuencia de los tratamientos fue aleatoria.

Medición de resultados principales

Evaluar y comparar los efectos de cada tratamiento sobre los lípidos y lipoproteinas, incluyendo la lipoproteina a [Lp(a)]

\section{Resultados Principales}

El análisis se realizó por intención de tratar*. Tanto la TRH como SMV redujeron de el colesterol total y el colesterol LDL, pero la SMV fue más efectiva (ver tabla). A diferencia de la SMV, la TRH producía un mayor aumento de la HDL cuanto más baja era ésta inicialmente. La reducción de la $\mathrm{Lp}(\mathrm{a})$ con TRH fue mayor cuanto mayor era la LDL basal; mientras que la SMV no alteró la Lp(a).

\begin{tabular}{|c|c|c|c|c|}
\hline 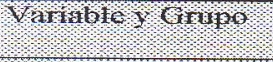 & 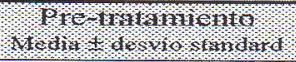 & 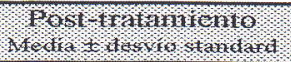 & 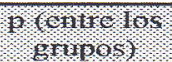 & 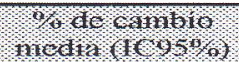 \\
\hline 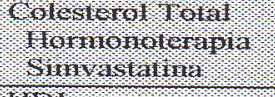 & $\begin{array}{l}305 \pm 41 \\
307 \pm 43 \\
\end{array}$ & $\begin{array}{l}255 \pm 32 \\
227 \pm 33 \\
\end{array}$ & $<0.001$ & $\begin{array}{l}-14(-16 a-11) \\
-26(-29 a-23)\end{array}$ \\
\hline 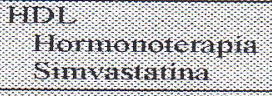 & $\begin{array}{l}63 \pm 17 \\
64 \pm 17 \\
\end{array}$ & $\begin{array}{l}67 \pm 16 \\
68 \pm 17 \\
\end{array}$ & 0.12 & $\begin{array}{l}+7(+2 a+12) \\
+7(+4 a+10)\end{array}$ \\
\hline 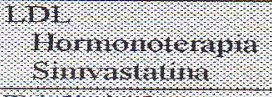 & $\begin{array}{l}212 \pm 400 \\
211 \pm 40\end{array}$ & $\begin{array}{l}154 \pm 29 \\
134 \pm 32 \\
\end{array}$ & $<0.001$ & $\begin{array}{l}-24(-28 \text { a }-20) \\
-36(-40 a-32) \\
\end{array}$ \\
\hline 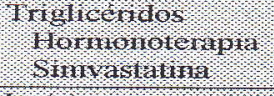 & $\begin{array}{l}151 \pm 60 \\
160 \pm 75 \\
\end{array}$ & $\begin{array}{l}172 \pm 59 \\
125 \pm 48\end{array}$ & $<0.001$ & $\begin{array}{c}+29(+15 a+42) \\
-14(-20 a-8) \\
\end{array}$ \\
\hline 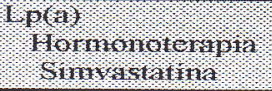 & $\begin{array}{l}34.5 \pm 38.6 \\
34.9 \pm 39.2\end{array}$ & $\begin{array}{l}23.8 \pm 25.3 \\
33.7 \pm 37.6\end{array}$ & 0.001 & $\begin{array}{l}21(-34 a-20) \\
+1(-6 a+8)\end{array}$ \\
\hline
\end{tabular}

Conclusiones:

La TRH es una terapia hipolipemiante efectiva en mujeres postmenopáusicas hipercolesterolémicas y puede ser una alternativa efectiva a la SMV, especialmente en mujeres con triglicéridos normales.

Fuente de Financiamiento: Elizabeth and Nicholas Slezak Trust

\section{Comentario}

Aunque aun no existe evidencia grado $I^{*}$ (estudios randomizados), hay importante evidencia grado II* que muestra que la hormonoterapia (TRH) reduce hasta en un $50 \%$ la incidencia y la mortalidad por enfermedad coronaria. Esto podría explicarse en gran parte por los efectos de la hormonoterapia en el perfil lipídico. Tanto éste como otros estudios randomizados y guías de práctica (1-2) confirman que la TRH debería ser el tratamiento de primera línea en mujeres postmenopáusicas con hipercolesterolemia. La TRH confiere además importantes beneficios para la prevención de osteoporosis, aunque aumenta con el uso crónico la incidencia de cáncer de mama (3). Otra ventaja importante con respecto a las estatinas como la simvastatina o pravastatina es el costo (costo anual TRH:\$240; estatinas:\$480-840). Estudios prospectivos sugieren que el riesgo coronario se reduce en un $2-3 \%$ por cada $\mathrm{mg} / \mathrm{dl}$ de ascenso de la HDL y aumenta un $1 \%$ por cada $\mathrm{mg} / \mathrm{dl}$ de ascenso de la LDL. La reducción de riesgo en base a estos datos sería similar para la TRH y las estatinas. A pesar de la alta dosis de estrógenos utilizada en este estudio, otros demuestran efectos similares con $0.625 \mathrm{mg}$ /día, y el estudio PEPI sugiere que la TRH cíclica es aun más beneficiosa (4). El aumento de los triglicéridos es consistente en todos los estudios y parece atenuarse con TRH combinada. Aunque aún no se sabe el impacto pronóstico del aumento de los triglicéridos, se debe tener cuidado con la TRH en mujeres con hipertrigliceridemia. Un hallazgo importante del presente estudio es que el beneficio de la TRH es mayor cuanto peor es el perfil lipídico de la paciente (a menor HDL, mayor aumento proporcional de HDL; y a mayor LDL, mayor descenso de Lp(a)). Esto podría implicar una mayor protección de la TRH en mujeres de alto riesgo cardiovascular.

*Ver Glosario

Dr. Federico Augustovshi Unidad de Medicina Familiar y Preventiva Hospital Italiano de Buenos Aires

\section{Referencias}

1. Davidson MH. Testolin LM. Maki KC y col. A comparison of estroge-t replacement, pravastatin, and combined treatment for the management of hypercholesterolemia in postmenopausal women. Arch Int Med 1997:157:1186-92

2. Summary of the II report of the National Cholesterol Education Pro gram (NCEP) expert panel on detection, evaluation and treatment of high blood cholesterol in adults. JAMA 1993:269:3015-23

3. Resumen de La terapia de reemplazo hormonal disminuye la mortal dad en las mujeres, en Evidencia en Atención Primaria. 1997 Octubre-Di. ciembre Vol 0 Nro 0: 5. Comentado de: Grodstein F, Stampfer MJ. Coldit GA et al. Postmenopausal homone therapy and mortality. N Engl J Med 1997:336:1769-75

4. The Postmenopausal Estrogen/Progestin Intervention Trial Writing Group Effects of estrogen or estrogen/progestin regimens on heart disease ris factors in postmenopausal women. JAMA 1995;273:199-208 